\title{
Thrombotic risk in COVID-19: a case series and case-control study
}

\author{
Authors: Simon M Stoneham, ${ }^{\mathrm{A}}$ Kate M Milne, ${ }^{\mathrm{B}}$ Elisabeth Nuttall,,${ }^{\mathrm{C}}$ Georgina H Frew, ${ }^{\mathrm{C}}$ Beattie RH Sturrock, ${ }^{\mathrm{D}}$ \\ Helena Sivaloganathan, ${ }^{\mathrm{E}}$ Eleni E Ladikou, ${ }^{\mathrm{E}}$ Stephen Drage, ${ }^{\mathrm{F}}$ Barbara Phillips, ${ }^{\mathrm{G}}$ Timothy $\mathrm{JT}$ Chevassut ${ }^{\mathrm{H}}$ and \\ Alice C Eziefula ${ }^{\mathrm{I}}$
}

\section{Background}

A possible association between COVID-19 infection and thrombosis, either as a direct consequence of the virus or as a complication of inflammation, is emerging in the literature. Data on the incidence of venous thromboembolism (VTE) are extremely limited.

\section{Methods}

We describe three cases of thromboembolism refractory to heparin treatment, the incidence of VTE in an inpatient cohort, and a case-control study to identify risk factors associated with VTE.

Results

We identified 274 confirmed (208) or probable (66) COVID-19 patients. 21 (7.7\%) were diagnosed with VTE. D-dimer was elevated in both cases (confirmed VTE) and controls (no confirmed VTE) but higher levels were seen in confirmed VTE cases (4.1 vs $1.2 \mu \mathrm{g} / \mathrm{mL}, \mathrm{p}<0.001$ ).

Conclusion

Incidence of VTE is high in patients hospitalised with COVID-19. Urgent clinical trials are needed to evaluate the

Authors: ${ }^{A}$ academic clinical fellow, Brighton and Sussex University Hospitals NHS Trust, Brighton, UK and Brighton and Sussex Medical School, Falmer, UK; ${ }^{B}$ academic clinical fellow, Brighton and Sussex Medical School, Brighton, UK and Royal Sussex County Hospital,

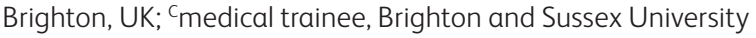
Hospitals NHS Trust, Brighton, UK; Dacademic foundation trainee, Brighton and Sussex Medical School, Brighton UK and Royal Sussex County Hospital, Brighton, UK; Eacademic clinical fellow, Brighton and Sussex University Hospitals NHS Trust, Brighton, UK and Brighton and Sussex Medical School, Falmer, UK; ${ }^{F}$ consultant in Intensive Care Medicine Brighton and Sussex University Hospitals NHS Trust, Brighton, UK; ${ }^{G}$ reader and consultant in intensive care medicine, Brighton and Sussex University Hospitals NHS Trust, Brighton, UK and Brighton and Sussex Medical School, Falmer, UK; ${ }^{H}$ reader in haematology and director of academic training, Brighton and Sussex Medical School, Brighton UK and consultant haematologist, Royal Sussex County Hospital, Brighton, UK; Isenior lecturer and consultant in infectious disease, Brighton and Sussex University Hospitals NHS Trust, Brighton, UK and Brighton and Sussex Medical School, Falmer, UK role of anticoagulation in COVID-19. Monitoring of D-dimer and anti-factor Xa levels may be beneficial in guiding management.

KEYWORDS: COVID-19, D-dimer, venous thromboembolism, pulmonary embolism, deep vein thrombosis

DOI: $10.7861 /$ clinmed.2020-0228

\section{Introduction}

Novel coronavirus SARS-CoV-2 emerged in late 2019 and has spread rapidly around the world. On 12 March 2020 the World Health Organisation declared a pandemic.' Severe COVID-19 infection may predispose individuals to thrombotic events or coagulopathy ${ }^{2-4}$ possibly mediated by excessive inflammation or direct virus effect. Poor outcomes have been observed in association with raised D-dimers, ${ }^{3,5}$ while anticoagulation may reduce disease mortality. ${ }^{6}$ Data on the incidence of venous thromboembolism (VTE) are currently limited.

\section{Key points}

What is known: High D-dimer levels are seen in patients with severe COVID-19 infection. Increased rates of venous thromboembolism (VTE) have been reported in COVID-19 patients admitted to critical care.

What is the question: What is the rate of VTE in hospitalised patients with COVID-19? What are the risk factors associated with VTE in hospitalised COVID-19 patients?

What was found: $21 / 274$ (7.7\%) COVID-19 patients were diagnosed with VTE. Most COVID-19 patients had elevated $(>0.5 \mu \mathrm{g} / \mathrm{mL}$ ) D-dimers (56/60, $93 \%$ ); however, levels were higher in patients with VTE (4.1 vs $1.2 \mu \mathrm{g} / \mathrm{mL}, p<0.001$ ).

Implications for practice: Clinicians should be vigilant for VTE in patients with COVID-19 infection and ensure prophylactic measures are in place. Very high D-dimer levels should increase clinical suspicion of venous thromboembolism. 
We identified an increased incidence of VTE among inpatients admitted with COVID-19. Additionally, several individuals appeared refractory to therapeutic anticoagulation with heparin. Based on these observations, we identified a consecutive series of hospital patients diagnosed with COVID-19 infection between 20 March 2020 and 9 April 2020 (274) and observed the rate of venous thromboembolic disease (21).

We describe the pertinent clinical features of three patients diagnosed with COVID-19 and venous thromboembolism who appeared refractory to initial treatment with heparin. A retrospective case-control design was used to identify significant clinical characteristics associated with VTE in patients admitted with COVID-19.

\section{Methods \\ Clinical setting}

This study was carried out at the Brighton and Sussex University Hospitals NHS Trust, including two acute hospital sites in southern England.

\section{COVID-19 data}

Patients were identified as having confirmed or probable COVID-19 if they had PCR-detected SARS-CoV-2 respiratory sample, or a chest $X$-ray (CXR) or computed tomography (CT) scan that was consistent with COVID-19 or probable COVID-19 according to a standardised imaging reporting protocol that was introduced on the 20 March 2020. Records for all positive SARS-CoV-2 virology between 20 March 2020 and 9 April 2020 were obtained from the laboratory inpatient management system (Winpath, Clinisys, Chertsey, UK). Records of all imaging reported as consistent with COVID-19 or probable COVID-19 between 20 March 2020 and 9 April 2020 were obtained from the picture archiving and communication system (Intellispace PACS, Philips, Amsterdam, NL).

\section{Clinical data}

Hospital admissions were identified using the patient administration system (Medway, System C, Maidstone, UK). For those patients admitted to hospital, admission blood test results were obtained from the laboratory inpatient management system (ICE, Sunquest, Tucson, US). Data on patients' past medical history were obtained by screening discharge summaries from the Patient Administration System (Medway, System C, Maidstone, UK). This system was also used to identify all patient deaths, of any cause, up to 18 April 2020. Records of all imaging reported as demonstrating VTE, including pulmonary embolism (PE) and deep vein thrombosis (DVT), from 20 March 2020 up to 16 April 2020 were obtained from the PACS. Only patients with radiologically confirmed VTE, including DVT and PE, were included. Data were collected on age, sex, critical care admission and comorbidities (history of cardiovascular, pulmonary, renal or malignant disease, diabetes, immunocompromise or immunosuppressive treatment). Admission blood results were collected for total white cell count, lymphocyte count, CRP, D-dimer, INR, APTT ratio and fibrinogen.

\section{Case-control study}

The case-control group was constructed at a ratio of 1:2, cases to controls. A case was defined as having imaging positive for VTE and either a respiratory tract sample PCR-positive for SARS-CoV-2 or imaging consistent with the diagnosis of COVID-19 pneumonia. Three cases did not have a D-dimer, APTT ratio or fibrinogen result recorded but had their other results included in the analysis. Control patients were identified from inpatients sequentially diagnosed either radiologically or via PCR without evidence of venous thromboembolism as of 16 April 2020. The ratio of PCRpositive to PCR-negative patients was matched between cases and controls. Control patients were excluded if clinical information was incomplete.

\section{Statistical analysis}

Statistical analysis was performed using Prism 8 (GraphPad Software, San Diego, US). Categorical variables were analysed by Z-test. Means of normally distributed continuous variables were compared by unpaired T-test. Means of non-normally distributed variables were compared by Mann-Whitney test. A logistic regression model was constructed to test the strength of association between clinical variables and the presence of venous thromboembolism. Results were considered significant if the p-value was $<0.05$.

\section{Ethics}

Informed consent was obtained from the patients in the case series. This work was carried out as a service evaluation. ${ }^{7}$ All data were anonymised for the purpose of analysis.

\section{Results}

Table 1 describes the clinical characteristics of three patients who were admitted with type 1 respiratory failure and diagnosed with COVID-19 infection. All three cases demonstrated persistent hypoxia and were subsequently diagnosed with VTE. Despite weight-based treatment with low molecular weight heparin, all patients demonstrated either sub-therapeutic anti-factor Xa levels or clinical progression of clot.

To determine the incidence of VTE among inpatients with COVID-19 pneumonia, we examined records of a consecutive series of inpatients with confirmed or possible COVID-19 infection from the 20 March 2020 and 9 April 2020. 274 hospital inpatients with confirmed or possible COVID-19 infection were identified. Of these, 208 out of 274 (76\%) patients had a diagnosis of COVID-19 confirmed by PCR on a respiratory specimen and 66 out of 274 (24\%) had a probable diagnosis, with imaging consistent with COVID-19. A total of 21 out of 274 (7.7\%) patients were diagnosed with venous thromboembolism up to 16 April 2020.16 out of 21 (76.2\%) patients were diagnosed with PE and 5 out of 21 (23.8\%) were diagnosed with DVT. We found a small but non-significant difference in the rate of VTE between patients with a confirmed diagnosis of COVID-19 and those with a probable (imaging confirmed) diagnosis of COVID-19 (14/208 [6.7\%] vs 7/66 [10.6\%], $p=0.07)$. As of 18 April 2020, the overall all-cause mortality rate in our cohort was 76 out of 274 (27.7\%). Significantly more deaths occurred in patients who were PCR-positive compared to those who were diagnosed radiologically (66/208 [31.7\%] vs 10/66 [15.2\%], $p=0.01$ ). However, outcome data are incomplete as not all patients have yet recovered fully from their disease.

21 patients with thromboembolism were identified as cases and 42 , without evidence of thromboembolism, identified as 
Table 1. Case series

\section{Characteristic}

Age

Comorbidities

Sex

Length of illness prior to

admission date (days)

Symptoms at onset

Imaging features

Clinical course

Critical care admission

VTE

\section{Laboratory findings on admission}

Positive PCR for SARS-

CoV-2

WCC $10^{9} / \mathrm{L}$

Lymphocyte count $10^{9} / \mathrm{L}$

CRP mg/L

367

1.2

INR

1.2

9.9

Fibrinogen $\mathrm{g} / \mathrm{L}$

D-dimer ug/ml FEU

5.19

\section{Special haematology}

Initial anti-Xa IU/ml

(0.4-1.0)

Repeat anti-Xa IU/ml

Antithrombin \%

(84-119)

Von Willebrand factor antigen \% (50-140)

Factor VIIIc u/dL (60-150)

\section{Case 2}

81

COPD, hypertension

Female

18

Dyspnoea, persistent cough, fever

Bilateral multifocal consolidation on CXR

Admitted with type 1 respiratory failure tachycardia and fever. Persistent tachycardic and hypoxic on day 3 of admission. A CTPA demonstrated extensive bilateral pulmonary emboli. Due to low anti-factor Xa levels despite treatment with tinzaparin (175 units $/ \mathrm{kg}$ ) she was switched to enoxaparin $1 \mathrm{mg} / \mathrm{kg}$ BD.

\section{$\mathrm{N}$}

PE

N

8

0.4

58

1.1

1

7.3

3.31

$-$

0.38

0.52

99

210

205

\section{Case 3}

60

Prostate cancer

Male

Dyspnoea

Bilateral ground glass opacification on CT

Intubated in emergency department due to profound hypoxia. On day 8 lower limb swelling noted. USS diagnosed common and superficial femoral vein thrombus. Pulmonary embolus on CTPA. Despite intravenous heparin the APTT ratio was subtherapeutic. Switched to argatroban and clinically improved to discharge from critical care.

Y

DVT and PE

Y

0.3

247

1.1

1.2

6.8

19.38

269

74

506

APTT = activated partial thromboplastin time; $C T P A=C T$ pulmonary angiogram; $C R P=C$-reactive protein; DVT $=$ deep vein thrombosis; INR $=$ international normalised ratio; $\mathrm{PE}=$ pulmonary embolism; $\mathrm{VTE}=$ venous thromboembolism. 
Table 2. Clinical characteristics of case-control sample

\begin{tabular}{|c|c|c|c|}
\hline Inpatients with COVID-19 & $\begin{array}{l}\text { VTE-positive } \\
(\mathrm{N}=21)\end{array}$ & $\begin{array}{l}\text { VTE-negative } \\
(\mathrm{N}=42)\end{array}$ & p-value \\
\hline Age $[\text { mean }(S D)]^{*}$ & $67(12)$ & $65(15)$ & 0.32 \\
\hline Female sex $[n(\%)]$ & $7(33)$ & $18(43)$ & 0.47 \\
\hline \multicolumn{4}{|l|}{ Comorbidities } \\
\hline Cardiovascular disease [n (\%)] & $6(29)$ & $9(21)$ & 0.53 \\
\hline Diabetes $[n(\%)]$ & $8(38)$ & $10(23)$ & 0.23 \\
\hline Pulmonary disease $[\mathrm{n}(\%)]$ & $8(38)$ & $9(21)$ & 0.16 \\
\hline Renal disease $[\mathrm{n}(\%)]$ & $4(19)$ & $6(14)$ & 0.63 \\
\hline Malignancy [n (\%)] & $4(19)$ & $6(14)$ & 0.63 \\
\hline Immunosuppression [n ( \% )] & $2(10)$ & $3(7)$ & 0.74 \\
\hline Admission to critical care [n ( \% )] & $6(29)$ & $14(33)$ & 0.70 \\
\hline \multicolumn{4}{|l|}{ Laboratory parameters } \\
\hline CRP mg/L [median (IQR)] & $122(188)$ & $78.5(101)$ & 0.09 \\
\hline White cell count $\times 10^{9} / L$ [median (IQR)] & $11.1(4.6)$ & $7.2(4.5)$ & 0.009 \\
\hline Lymphocytes $\times 10^{9} / \mathrm{L}$ [median (IQR)] & $0.9(0.7)$ & $0.9(0.6)$ & 0.95 \\
\hline D-dimer $\mu \mathrm{g} / \mathrm{mL}$ [median (IQR)] & $4.1(6.0)$ & $1.2(2.0)$ & $<0.001$ \\
\hline APTT ratio [median (IQR)] & $1.1(0.1)$ & $1.2(0.3)$ & 0.72 \\
\hline Fibrinogen g/L [mean (SD)] & $6.8(2.2)$ & $6.6(1.8)$ & 0.7 \\
\hline INR [median (IQR)] & $1.1(0.2)$ & $1.05(0.1)$ & 0.02 \\
\hline
\end{tabular}

Sequentially diagnosed patients without VTE diagnosis as of 9 April 2020 were selected as controls at a ratio of 2:1. Three cases without measurements of D-dimer, APTT ratio or fibrinogen were included in the analysis. Control patients were excluded if data was incomplete. Control patients were selected at a ratio of 2:1 swab positive (28) to swab negative (14) to match the distribution of cases. D-dimer above 20 was recorded as 20 for the purposes of analysis. Normally distributed data is marked with an asterisk (*). Proportions were analysed by Z-test, normally distributed variables by unpaired T-test and non-parametric variables by Mann-Whitney test. APTT = activated partial thromboplastin time; CRP = C-reactive protein; INR = international normalised ratio; IQR = interquartile range; SD = standard deviation; VTE = venous thromboembolism.

controls. Their clinical features are summarised in Table 2. We found significant differences in levels of D-dimer (4.1 vs $1.2 \mu \mathrm{g} / \mathrm{ml}$, $p<0.001)$ and white cell count (11.1 vs $7.2 \times 10^{9} / \mathrm{L}, p=0.009$ ). There was a small, but statistically significant, difference in baseline INR (1.1 vs 1.05, $p=0.02$ ). There were no significant differences in age, gender or presence of comorbidities between the two groups.

Using multiple logistic regression modelling with adjustment for age, sex and SARS-CoV-2 swab PCR-positivity, we found a significant association between white cell count (odds ratio [OR] 1.18, $p=0.03$ ) $D$-dimer (OR 1.39, $p=0.004$ ) and fibrinogen (OR $1.66, p=0.03$ ) with the occurrence of VTE in COVID-19 patients (Table 3). Although the OR of VTE appeared higher for female sex (3.55), it was not statistically significant $(p=0.12)$.

To further examine the positive association between D-dimer and VTE, we stratified patients according to D-dimer result (Table 4). Almost all patients had an abnormal D-dimer result at baseline, defined as a D-dimer $>0.5 \mu \mathrm{g} / \mathrm{mL}(17 / 18,94 \%$ VTE positive patients vs 39/42 VTE negative patients, OR 1.3 p=0.82); however, a D-dimer threshold of $>2 \mu \mathrm{g} / \mathrm{mL}$ was present in $14 / 18$ (78\%) of patients with VTE and only 14/42 (33\%) patients without VTE (OR 7, p=0.002).
Table 3. Multivariate analysis using logistic regression for factors associated with the presence of VTE

$\begin{array}{lll}\text { Predictor variables } & \text { Odds ratio }(95 \% \text { CI }) & \text { p-value } \\ \text { Age } & 0.96(0.91-1.02) & 0.22 \\ \text { Female sex } & 3.55(0.78-19.6) & 0.12 \\ \text { White cell count } & 1.18(1.02-1.40) & 0.03 \\ \left(\times 10^{9} / \mathrm{L}\right) & & \\ \text { D-dimer }(\mu \mathrm{g} / \mathrm{mL}) & 1.39(1.15-1.84) & 0.004 \\ \text { Fibrinogen }(\mathrm{g} / \mathrm{L}) & 1.66(1.07-2.73) & 0.03 \\ \text { SARS-CoV-2 } & 0.98(0.23-4.59) & 0.98 \\ \text { PCR-positive } & & \end{array}$

Non-significant associations were removed. Significant associations were adjusted for age, sex and PCR positivity. 
Table 4. Stratified univariate analysis using pragmatic D-dimer cut-offs

\begin{tabular}{lllll}
\hline D-dimer threshold $(\boldsymbol{\mu g} / \mathbf{m L})$ & VTE + & VTE- & Odds ratio (95\% CI) & p-value \\
D-dimer $>0.5$ & $17 / 18(94 \%)$ & $39 / 42(92 \%)$ & $1.3(0.18-17.9)$ & 0.82 \\
D-dimer $>1$ & $17 / 18(94 \%)$ & $27 / 42(64 \%)$ & $9.4(1.41-105)$ & 0.02 \\
D-dimer $>2$ & $14 / 18(78 \%)$ & $14 / 42(33 \%)$ & $7.0(1.95-21.6)$ & 0.002 \\
D-dimer $>3$ & $12 / 18(67 \%)$ & $10 / 42(24 \%)$ & $6.4(1.87-19.3)$ & 0.002 \\
D-dimer $>4$ & $9 / 18(50 \%)$ & $8 / 42(19 \%)$ & $4.3(1.32-14.2)$ & 0.01 \\
\hline Statistical significance was determined by Chi-squared test. VTE = venous thromboembolism.
\end{tabular}

\section{Discussion}

A number of observational studies have alluded to the presence of coagulopathy in severe COVID-19 patients, including abnormal clotting parameters and the presence of overt DIC. ${ }^{2-5} \mathrm{~A}$ recent pooled analysis by Lippi and Favaloro highlighted an association between D-dimer and severe COVID-19 infection. ${ }^{5}$ Tang and colleagues described an association between heparin use and survival in patients with a high 'sepsis-induced coagulopathy' (SIC) score or D-dimer. ${ }^{6}$ Despite these observations, reports of thrombotic complications are limited. Klok and colleagues report on the incidence of thrombotic complications (both arterial and venous) in critical care patients in two Dutch university hospitals, finding an overall rate of $31 \%{ }^{8}$ Cui et al report a DVT incidence of $25 \%$ among 81 intensive care patients. ${ }^{9}$ The data described here support previous findings, that D-dimer is markedly elevated in patients with COVID-19 infection, and this study provides the first peer-reviewed quantitative analysis of venous thromboembolism including adult patients with COVID-19 infection outside of an intensive care setting. We highlight the possibility that some individuals infected with COVID-19 may be refractory to treatment with low molecular weight heparin. Reports of the use of thrombolysis in these patients remain scanty within the literature. ${ }^{10}$

Our analysis included patients with probable COVID-19 infection based on radiological findings. We found that 66 out of 274 (24.1\%) of our patients were PCR-negative on upper respiratory tract samples but had a clinical syndrome consistent with COVID-19 infection. Previous authors have observed that clinical deterioration often occurs late in disease, ${ }^{4}$ at this time, upper respiratory tract samples may be negative. ${ }^{11,12}$ Consistent with these findings we saw a tendency towards higher rates of VTE in patients who were PCR-negative, although the differences were not statistically significant and there was no significant association between PCR status and the presence of VTE in the multivariate analysis (Table 3). It should be noted that there is a difference in mortality between those with radiologically diagnosed COVID-19 and those who were diagnosed by PCR. It is possible some of the patients in the radiology arm had an alternative diagnosis. It is difficult to speculate on the mortality difference due to the incomplete period of follow-up.

D-dimer, a breakdown product of fibrin, is elevated in the acute phase of the inflammatory response. ${ }^{13}$ What remains unclear is whether rate of VTE described here is attributable specifically to COVID-19 infection or, alternatively, is representative of patients with a profound inflammatory response. Severe COVID-19 infection has been associated with markedly elevated inflammatory markers. $2,4,13,15,16$ We found significantly higher levels of circulating white cells and fibrinogen, but not C-reactive protein (CRP), in patients who developed VTE compared to those with no VTE (Tables 2 and 3). Outside of COVID-19 infection, several authors have described an association between thrombotic risk and circulating levels of inflammatory markers including CRP and pro-inflammatory cytokines, notably IL- 6 and IL-8, ${ }^{16-18}$ although data in acute inflammation are lacking. Given our small sample size, it is possible our study was underpowered to detect differences in CRP. Notably high rates of thromboembolism are seen with ARDS and severe pulmonary infection. ${ }^{19}$ Data on incidence of venous thromboembolism with specific viral respiratory infections is limited. However, one case series identified a rate of $3.4 \%$ among 119 hospitalised patients with $\mathrm{H} 1 \mathrm{~N} 1$ influenza. $^{20}$

The role of therapeutic anticoagulation, particularly in ARDS and severe sepsis, continues to be debated. ${ }^{19,21-24}$ Blockade of the ACE2 receptor, the receptor by which SARS-CoV-2 enters cells via S-spike binding, ${ }^{25}$ has been associated with inflammatory effects. ${ }^{26}$ ACE2 is expressed on vascular endothelium. ${ }^{27}$ In the case series, levels of von Willebrand factor (VWF) and factor VIII were extremely high. This may be attributable to viral disruption of the endothelium leading to release of vWF from the Weibel-Palade storage bodies and exposing underlying collagen with release of prothrombotic mediators resulting in increased fibrin turnover and hypercoagulability. This requires further investigation.

Overall, these data confirm that D-dimer levels are raised in most hospitalised patients with COVID-19 infection and may be associated with increased rates of venous thromboembolism. Although rates of thromboembolism were high in this series, we suspect overall rates of VTE may be under-diagnosed in COVID-19 pneumonia. Within our hospitals we have introduced D-dimer levels as a routine investigation in all suspected COVID-19 patients to support the detection of VTE. In addition, we are measuring anti-factor Xa levels in patients diagnosed with VTE on treatment dose anticoagulation to ensure therapeutic dosing. Urgent clinical trials are required to investigate the role of enhanced prophylactic and therapeutic anticoagulation in these patients as a way of abrogating the high levels of mortality seen in hospitalised COVID-19 patients.

\section{Acknowledgements}

The authors would like to acknowledge Dr Ting Ting Zhang for her assistance obtaining radiology data. The authors would also like to acknowledge Lenka Mercer for her assistance in laboratory analysis. 


\section{References}

1 World Health Organisation. Health topics (2020). Available from www.euro.who.int/en/health-topics/health-emergencies/coronavirus-covid-19/news/news/2020/3/who-announces-covid-19-outbreak-a-pandemic [Accessed 18 April 2020].

2 Chen Tao, Wu Di, Chen Huilong et al. Clinical characteristics of 113 deceased patients with coronavirus disease 2019: retrospective study. BMJ 2020;368:m1091.

3 Tang N, Li D, Wang X, Sun Z. Abnormal coagulation parameters are associated with poor prognosis in patients with novel coronavirus pneumonia. J Thromb Haemost 2020;18:844-7.

4 Zhou F, Yu T, Du R et al. Clinical course and risk factors for mortality of adult inpatients with COVID-19 in Wuhan, China: a retrospective cohort study. Lancet 2020;395:1054-62.

5 Lippi G, Favaloro EJ. D-dimer is associated with severity of coronavirus disease 2019: a pooled analysis. Thromb Haemost 2020;120:876-8.

6 Tang N, Bai H, Chen X et al. Anticoagulant treatment is associated with decreased mortality in severe coronavirus disease 2019 patients with coagulopathy. J Thromb Haemost 2020;18:1094-99.

7 Health Research Authority. Defining research table. HRA, 2017. Available from www.hra-decisiontools.org.uk/research/docs/ DefiningResearchTable_Oct2017-1.pdf [Accessed 16 April 2020].

8 Klok FA, Kruip MJHA, van der Meer NJM et al. Incidence of thrombotic complications in critically ill ICU patients with COVID-19. Thromb Res 2020;pii:S0049-3848(20)30120-1.

9 Cui S, Chen S, Li X, Liu S, Wang F. Prevalence of venous thromboembolism in patients with severe novel coronavirus pneumonia. J Thromb Haemost 2020, in press (doi: 10.1111/jth.14830).

10 Wang J, Hajizadeh N, Moore EE et al. Tissue plasminogen activator (tPA) treatment for COVID-19 associated acute respiratory distress syndrome (ARDS): a case series. J Thromb Haemost 2020, in press (doi: 10.1111/jth.14828).

11 Zou L, Ruan F, Huang M et al. SARS-CoV-2 viral load in upper respiratory specimens of infected patients. N Engl J Med 2020;382:1177-9.

12 Yang Y, Yang M, Shen C et al. Evaluating the accuracy of different respiratory specimens in the laboratory diagnosis and monitoring the viral shedding of 2019-nCoV infections. MedRxiv 2020.02.11. 20021493.

13 Lippi G, Bonfanti L, Saccenti C, Cervellin G. Causes of elevated D-dimer in patients admitted to a large urban emergency department. Eur J Intern Med 2014;25:45-8.

14 Henry BM, de Oliveira MHS, Benoit S, Plebani M, Lippi G. Hematologic, biochemical and immune biomarker abnormalities associated with severe illness and mortality in coronavirus disease 2019 (COVID-19): a meta-analysis. Clin Chem Lab Med 2020;pii:/j/ cclm.ahead-of-print/cclm-2020-0369/cclm-2020-0369.xml.

15 Lippi G, Plebani M. Procalcitonin in patients with severe coronavirus disease 2019 (COVID-19): a meta-analysis. Clin Chim Acta 2020; 505:190-1.

16 Folsom AR, Lutsey PL, Astor BC, Cushman M. C-reactive protein and venous thromboembolism. A prospective investigation in the ARIC cohort. Thromb Haemost 2009;102:615-9.

17 Saghazadeh A, Rezaei N. Inflammation as a cause of venous thromboembolism. Crit Rev Oncol Hematol 2016;99:272-285.

18 Matos MF, Lourenço DM, Orikaza CM et al. The role of IL-6, IL-8 and MCP-1 and their promoter polymorphisms IL-6 -174GC, IL-8 -251AT and MCP-1 -2518AG in the risk of venous thromboembolism: a case-control study. Thromb Res 2011;128:216-20.

19 Frantzeskaki F, Armaganidis A, Orfanos SE. Immunothrombosis in acute respiratory distress syndrome: cross talks between inflammation and coagulation. Respiration 2017:93:212-25.

20 Bunce PE, High SM, Nadjafi M. Pandemic H1N1 influenza infection and vascular thrombosis. Clin Infect Dis 2011;52:e14-e17.

21 Ryan D, Frohlich S, McLoughlin P. Pulmonary vascular dysfunction in ARDS. Ann Intensive Care 2014;4:28.

22 Allingstrup M, Wetterslev J, Ravn FB, Møller AM, Afshari A. Antithrombin III for critically ill patients. Cochrane Database Syst Rev 2016;2:CD005370.

23 Camprubí-Rimblas M, Tantinyà N, Bringué J, Guillamat-Prats R, Artigas A. Anticoagulant therapy in acute respiratory distress syndrome. Ann Transl Med 2018;6:36.

24 Ranieri VM, Thompson BT, Barie PS et al. Drotrecogin alfa (activated) in adults with septic shock. N Engl J Med 2012;366:2055-64.

25 Yan R, Zhang Y, Li Y, Xia L, Guo Y, Zhou Q. Structural basis for the recognition of SARS-CoV-2 by full-length human ACE2. Science 2020;367:1444-48.

26 Kuba K, Imai Y, Penninger JM. Angiotensin-converting enzyme 2 in lung diseases. Curr Opin Pharmacol 2006;6:271-6.

27 Hamming I, Timens W, Bulthuis ML et al. Tissue distribution of ACE2 protein, the functional receptor for SARS coronavirus. A first step in understanding SARS pathogenesis. J Pathol 2004;203:631-7.

Address for correspondence: Dr Simon Stoneham, Department of Microbiology and Infection, Royal Sussex County Hospital, Eastern Road, Brighton, Sussex BN2 5BE, UK.

Email: s.stoneham@bsms.ac.uk 